\title{
HACIA UN DICCIONARIO ELECTRÓNICO DE FRASEOLOGÍA BILINGÜE EN ESPAÑOL Y CATALÁN: REFLEXIÓN EN TORNO A SUS FUNCIONES Y LAS PERCEPCIONES DE LOS USUARIOS ${ }^{1}$
}

\author{
TOWARDS AN ELECTRONIC DICTIONARY OF BILINGUAL \\ PHRASEOLOGY IN SPANISH AND CATALAN: REFLECTION \\ ABOUT ITS FUNCTIONS AND THE PERCEPTIONS OF USERS
}

\section{JOSEPH GARCÍA RODRÍGUEZ \\ Universidad Autónoma de Barcelona \\ joseph.garcia@uab.cat}

\section{RESUMEN}

Los diccionarios bilingües en papel y en formato electrónico son una herramienta de gran ayuda para todos los que trabajan con dos lenguas. No obstante, la concepción de estas obras no siempre se ha basado en los avances que se han producido en la lexicografía. El problema es mayor cuando se trata de elaborar un diccionario bilingüe específico, como lo es aquel que solo registra unidades fraseológicas (UFS). Ante un proyecto lexicográfico de este tipo, pocos son los autores que recogen la opinión de los usuarios emergentes con el fin de ceñir el diseño a sus necesidades. Los objetivos principales de la presente investigación son, por un lado, analizar los puntos fuertes y débiles que, según los usuarios, poseen estas obras de consulta y, por otro, reflexionar en torno a cómo debería elaborarse una obra fraseográfica bilingüe en formato electrónico, dado que no existe ninguna de este tipo en el contexto español-catalán.

Palabras clave: Fraseografía bilingüe, diccionarios electrónicos, encuestas.

\footnotetext{
${ }^{1}$ El desarrollo de esta investigación ha sido posible gracias a la ayuda del Ministerio de Economía y Competitividad para el proyecto «El español de Cataluña en los medios de comunicación orales y escritos» (FFI2016-76118-P) y al apoyo de la AGAUR de la Generalitat de Catalunya para el «Grup de Lexicografia i Diacronia» (2017 SGR 1251).
} 


\begin{abstract}
Bilingual dictionaries on paper and in electronic format are an extremely helpful tool for all those working with two languages. However, its conception has not always been based on the advances that have occurred in lexicography. The problem is greater when it comes to developing a specific bilingual dictionary, such as one that only includes phraseological units (PUS). Faced with a lexicographic project of this type, few authors collect the opinion of emerging users in order to narrow the design to their needs. In this research, the main objectives are, on the one hand, to analyse the strengths and weaknesses that, according to users, identify these dictionaries and, on the other hand, how we should prepare a bilingual phraseological dictionary in electronic format, since none of this type exists in the Spanish-Catalan context.
\end{abstract}

Keywords: Bilingual phraseography, electronic dictionaries, surveys.

Recibido: 06/04/2020. Aceptado: 21/05/2020.

\title{
1. LA NECESIDAD DE ELABORAR DICCIONARIOS ELECTRÓNICOS
}

T a fraseología bilingüe en español y catalán, tanto desde el punto de vista teóLrico como práctico, es todavía hoy un reto para los investigadores. Aunque el desarrollo de la lexicografía electrónica aún se encuentra en una fase inicial, sobre todo para las obras bilingües, es necesario plantear propuestas que permitan un avance en este ámbito, puesto que los usuarios prefieren, cada vez más, diccionarios en línea que concedan una búsqueda rápida y sencilla de la información. Aun así, en determinados contextos, como en la enseñanza, todavía hay profesionales que evitan o no recomiendan el uso de este tipo de obras por considerarlas poco fiables, debido al desconocimiento y a la creencia de que las aplicaciones en línea carecen de rigor científico (Sala Caja, 2015). No obstante, la irrupción de las nuevas tecnologías y su extensión entre los más jóvenes han obligado a determinados sectores a formarse en esta materia y a aceptar que no todo lo que se encuentra en línea carece de calidad.

El desarrollo de la lingüística computacional ha permitido reorientar el rumbo de los estudios y sus aplicaciones en la lexicografía electrónica, dado que el diccionario no debe ser un mero repertorio de equivalentes, sino que debe estar enfocado a solventar las dudas desde diferentes perspectivas de las personas que lo consultan. No basta con priorizar el contenido; es importante reflexionar en torno a los tipos de búsqueda que se deben proponer para que el usuario obtenga de manera exitosa la información que desea.

El formato en papel no siempre cumple con estos objetivos. En la década de los 90, algunos investigadores, como Meijs (1990: 69-70), se aventuraban a presagiar 
los cambios que se iban a producir en este ámbito: «I would not be surprised if for most general uses dictionaries in book-form will be antiquated before the end of the century». Esto demuestra que ya desde hace años los especialistas consideran pertinente una actualización del modo en que estos se conciben² ${ }^{2}$.

En consonancia con lo anterior, algunos diccionarios en formato papel no solo se han visto limitados por la falta de espacio, con las consecuencias que esto conlleva en cuanto al tratamiento de la información, sino que, en ocasiones, la finalidad con la que se han elaborado ha sido puramente editorial. Todo esto permite afirmar que las obras lexicográficas en general, ya sean en formato papel o electrónico, necesitan una revisión teórico-práctica que ayude a conocer sus puntos fuertes y débiles, con el propósito de aplicar los resultados a la elaboración de nuevas obras en formato electrónico.

Algunas investigaciones como la de Müller-Spitzer et al. (2011) han demostrado cuáles son las características más relevantes que deben contener estos diccionarios, según una encuesta llevada a cabo para quienes consultan este tipo de obras (traductores, lingüistas y público en general). El estudio revela, sobre todo, las cuatro condiciones que los usuarios reconocen como esenciales en la creación de cualquier diccionario:

(1) La confiabilidad y la veracidad de la información que se aporta.

(2) La claridad con la que se muestran los datos.

(3) La posibilidad de actualizar la obra continuamente.

(4) La rapidez de la consulta.

Estos rasgos serán objeto de análisis, también, en la encuesta presentada en este artículo.

\section{LA FRASEOGRAFÍA EN ESPAÑOL Y CATALÁN: PUNTO DE PARTIDA}

En el contexto español-catalán parece evidente la necesidad de elaborar un diccionario con todas estas características, puesto que actualmente no existe una obra que recoja los avances en las investigaciones lexicográficas en formato electrónico. Del mismo modo, tampoco se encuentra un diccionario fraseológico en papel que, además del equivalente en la otra lengua, proporcione información completa y de posible interés para el usuario, sobre todo cuando el equivalente propuesto

\footnotetext{
${ }^{2}$ Véase García Rodríguez (2016) para conocer cómo ha evolucionado el tratamiento de la fraseología en algunos diccionarios bilingües generales.
} 
contiene matices distintos a la UF de la lengua de origen ${ }^{3}$. Asimismo, al trabajar con dos idiomas que forman parte de la misma familia lingüística, es interesante incluir datos que manifiesten las semejanzas y/o diferencias entre sus fraseologismos con el fin de ayudar al interesado a hacer un uso más adecuado de cada una de las unidades según el contexto comunicativo en que se halle. Mediante estudios contrastivos, como las de García Rodríguez (en prensa 2019 y 2020) en el contexto español-catalán, es posible conocer las características concretas de cada sistema lingüístico con el que se trabaja. Este modo de proceder es indispensable para evitar los problemas que se evidencian en los diccionarios cuando se proponen los equivalentes de las UFS 4 .

Como ejemplo de lo que se acaba de exponer, a pesar de que la fraseología de dos lenguas emparentadas, como lo son el español y el catalán, suele presentar semejanzas evidentes, también es común observar diferencias notables que se deben conocer para evitar calcos o interferencias entre los dos idiomas. Las disimilitudes que se pueden hallar entre las UFS de las lenguas aquí concernientes son de distinto tipo según los cambios que se producen en los componentes que las forman: parciales (echar fuego por los ojos - treure foc pels queixals; como pez en el agua - com el peix a l'aigua) o totales (darle aire - separar-se del bou; sacar agua de las piedras - treure foc). En las primeras se detectan escasas alteraciones léxicas o sintácticas, mientras que en las segundas los constituyentes de ambos fraseologismos son distintos. Incluso, en el caso de darle aire - separar-se del bou, el equivalente en catalán no es una UF, sino una paráfrasis, puesto que en dicho idioma no se encuentra una expresión relacionada con la tauromaquia para hacer referencia a 'separarse el torero del toro que muestra señales de debilidad y permitirle descansar unos momentos durante la faena de muleta' (GDUEsA, s. v. aire). Por el contrario, para sacar agua de las piedras sí que existe un equivalente fraseológico en catalán (treure foc), aunque este no se corresponda con la forma de la locución española. Ambos significan 'conseguir beneficios hasta de lo más difícil' (DFDEA, s. v. agua). Asimismo, es posible hallar unidades que, además de modificar algunos de sus elementos léxicos, también alteran el orden de sus componentes por el hecho de estar fijados de

${ }^{3}$ El diccionario bilingüe se concibe como una obra donde la prioridad es mostrar el equivalente en la lengua de destino; sin embargo, el formato electrónico rompe esta barrera y permite la inclusión de otro tipo de información (visible o no, según cómo esté planteada la estructura del diccionario y las necesidades que posea el usuario) hasta ahora impensable por las limitaciones que conlleva una obra en papel.

${ }^{4}$ Es necesario destacar la diferencia entre fraseología y fraseografía. El primer término alude a la disciplina lingüística que se encarga de estudiar las expresiones (unidades fraseológicas o fraseologismos) formadas por dos o más palabras separadas en la escritura, cuyas características fundamentales son la pluriverbalidad, la lexicalización, la institucionalización, la fijación y la idiomaticidad en cierto grado. El segundo se refiere a la «disciplina que se dedica a elaborar y poner en práctica los principios de tratamiento de las unidades fraseológicas en los diccionarios generales y fraseológicos. La fraseografía se dedica a recoger y registrar el conjunto (o una parte) de los fraseologismos de una lengua (o varias lenguas) [...]» (Corpas Pastor y Morvay, 2002: 166). 
una manera concreta en la lengua a la que pertenecen: dos aguas de abril y una de mayo valen bueyes y el carro - una pluja de maig $i$ tres d'abril valen els bous $i$ el carril.

Con todo, es imprescindible proponer un diccionario electrónico de fraseología bilingüe en estos dos idiomas para que los usuarios puedan acceder a este tipo de información y, de este modo, evitar traducciones literales o interferencias entre las UFS del español y el catalán y superar algunas de las deficiencias encontradas en las obras actuales.

El objetivo principal que se debería perseguir es el de poner a disposición de los destinatarios una obra que sirva como fuente de consulta fundamental para el conocimiento de ambos idiomas. No solo hacer hincapié en cómo se traduce un fraseologismo a la otra lengua, sino también enriquecer la competencia comunicativa de la persona que realiza la consulta, mediante informaciones que le ayuden a entender el significado de los fraseologismos y su uso. Asimismo, el hecho de que el español y el catalán puedan disponer de un diccionario fraseológico con estos rasgos conllevaría, seguramente, la proliferación de otros estudios relacionados con la lexicografía. Esto permitiría compensar la escasa producción de investigaciones centradas en la fraseología bilingüe y los diccionarios electrónicos entre estos dos idiomas, en comparación con los estudios disponibles actualmente en otros contextos.

\section{PROBLEMAS QUE PRESENTA UNA OBRA LEXICOGRÁFICA DE ESTE TIPO}

El lexicógrafo, cuando se dispone a preparar un diccionario, se enfrenta a problemas que no siempre tienen fácil solución. Elaborar una obra exclusivamente de fraseología entraña ciertas dificultades como, por ejemplo, decidir qué UFS se van a incluir y cómo se van a presentar al usuario de manera que el resultado de las búsquedas sea satisfactorio y suficiente para cumplir sus expectativas. Algunos de los diccionarios fraseológicos ya publicados muestran de manera explícita la sensación de inseguridad que conlleva la elaboración de una obra con estas características: «la compilación de un diccionario fraseológico es siempre una aventura llena de dificultades» (DFDEA, 2004: XI).

A diferencia de lo que ocurre con el formato en papel, el electrónico posee más libertad en cuanto a espacio y cantidad de información recogida, debido a que al no ser un producto físico puede contener más datos sin que estos supongan un obstáculo. Asimismo, al no tratarse de un producto comercial, ya que puede consultarse en línea de manera gratuita o mediante un registro que requiera un pago mensual o anual, el usuario puede acceder a él con más facilidad. No obstante, para poder crear este formato se necesitan recursos informáticos y, por consiguiente, económicos que complican su confección. Es decir, para poder aunar el cono- 
cimiento lingüístico con la tecnología resulta indiscutible la ayuda de un experto en informática (ingenieros o técnicos especializados, entre otros), que materialice la obra ideada por el lexicógrafo. Dicha labor no siempre es posible, puesto que el factor económico es un problema que determina su ejecución, sobre todo cuando se trata de proyectos no financiados o con pocos recursos personales.

Las dificultades aumentan cuando se pretende elaborar una obra lexicográfica en la que se plasmen los rasgos propios y comunes de la fraseología de dos lenguas. En el caso del español y el catalán, al tratarse de dos idiomas cercanos que pertenecen a la misma familia, un diccionario con estas características podría ayudar a solventar dudas relacionadas con las especificidades que presentan los fraseologismos en cada una de las dos lenguas. En determinados diccionarios bilingües generales (no fraseológicos) se menciona la necesidad de poseer los recursos necesarios para que el usuario pueda conocer los rasgos propios de cada idioma y, así, evitar los calcos:

[...] si la característica más evidente de nuestro panorama sociolingüístico es la existencia de dos lenguas en contacto, todo cuanto contribuya a conocerlas, a delimitar con precisión sus contenidos, su alcance y sus confines, a comparar sus respectivas estructuras gramaticales, a evitar, en fin, los calcos y las confusiones, constituye, indudablemente, una herramienta preciosa e imprescindible (DCC, 2006: s. p.).

El fet que les situacions de contacte entre aquestes llengües siguin quotidianes i generals en la major part del territori de parla catalana exigeix disposar d'uns instruments cada vegada més eficaços per donar resposta a les consultes dels usuaris (D62, 2003: s. p.).

En estas obras generales también se hace referencia a las UFS como expresiones que suelen acarrear ciertas dificultades para los hablantes, ya que al estar fijadas en la lengua dependen, en parte, del conocimiento específico de cada idioma.

Aquest diccionari els estalviarà de fer "castellanades" (mot que, segons el Fabra, és "dit d'una falta greu de castellà comesa especialment per un català en parlar o escriure una llengua"). Penso sobretot en les castellanades de locucions i de frases fetes, que són les més subtils (DCC, 2006: s. p.).

[...] hi ha barbarismes completament innecessaris perquè la nostra llengua posseeix els mots respectius en el patrimoni idiomàtic propi. I aquests són els barbarismes que cal evitar i que ja no han d'aparèixer en un diccionari que vulgui ésser almenys orientador, si no pròpiament normatiu. (Moll, 1980: XIV). 
A diferencia de estos diccionarios generales, en los que se recogen UFS no se encuentran referencias relacionadas con estas dificultades, lo que parece curioso si se tiene en cuenta que donde los usuarios suelen hallar más problemas es en la fraseología, dado que, como ya se ha comentado anteriormente, estas expresiones presentan características concretas en cada una de las lenguas y no siempre es posible traducirlas por un equivalente total.

\section{ENCUESTA SOBRE LA ELABORACIÓN DE UN DICCIONARIO ELECTRÓNICO}

Un diccionario debe ser un producto útil para las personas que lo consultan. A diferencia de aquellas obras que solo siguen criterios puramente comerciales, el diccionario que se desarrollará en futuras investigaciones pretende responder a las necesidades de los usuarios para que puedan encontrar en él la solución a determinadas dudas lingüísticas.

Para conseguir dicho objetivo, se ha elaborado una breve encuesta basada en preguntas relacionadas con el uso, el formato, el contenido y las características de dicha obra (véase el Anexo), con el fin de conocer cuáles son las prioridades de las personas que pueden llegar a convertirse en usuarios potenciales del diccionario. Así pues, la obra en cuestión tomará como base la experiencia y las necesidades reales de un grupo representativo de personas que forman parte de diferentes sectores.

Algunos autores ya han señalado con anterioridad la necesidad de llevar a cabo análisis prácticos que permitan dar cuenta del estado en que se encuentra el objeto de estudio con el que se pretende trabajar. Entre los métodos más empleados por los especialistas destacan las encuestas, útiles para detectar los intereses de los usuarios (Azorín, 2000a y 2000b; Climent de Benito, 2003), o el análisis de ciertas evidencias, las cuales ayudan a obtener una muestra representativa del ámbito que se pretende analizar. A modo de ejemplo, es necesario resaltar la labor desarrollada por Prat Sabater (2015), quien utiliza un corpus de escritos con el propósito de analizar las posibles interferencias lingüísticas que producen los estudiantes universitarios bilingües en el contexto español-catalán.

En el presente artículo, el método que se emplea se basa en recopilar información acerca de los participantes, no solo desde el punto de vista del uso que le otorgarían al diccionario en sí, sino también teniendo en consideración los datos personales que permiten comprobar la implicación más o menos directa que puede tener la persona con el objetivo de la investigación desarrollada.

Por consiguiente, se han diferenciado cinco grandes bloques. Cada uno de ellos contiene una o varias preguntas (véase el Anexo para consultar la encuesta). 
Los detalles y resultados se irán mostrando a continuación:

(1) Formación.

(2) Datos personales: edad, género, lugar de nacimiento y lugar de residencia habitual.

(3) Uso de las lenguas.

(4) Uso de los diccionarios bilingües en general.

(5) Posibles usos y funciones del futuro diccionario fraseológico.

Relacionado con (1), teniendo en cuenta que la obra pretende ser útil tanto para los usuarios especializados como para aquellos que solo quieran realizar una búsqueda simple para solventar una determinada duda, las personas que han respondido el formulario se encuentran cursando un grado universitario donde la lengua posee un papel esencial: Educación, Filología, Humanidades y Traducción. Sería conveniente, por tanto, ampliar dicha información en futuras investigaciones para poder conocer los intereses de los usuarios no especializados, así como de los que ya poseen una titulación superior y se encuentran en el mercado laboral. Sin embargo, en este artículo se ha apostado por realizar la encuesta a estudiantes de primer año de grado por considerar que se encuentran a caballo entre la educación secundaria y la universidad, y esto les permite valorar desde diferentes puntos de vista las preguntas llevadas a cabo en este formulario.

En total se han obtenido 147 respuestas, divididas según la formación que se está cursando:

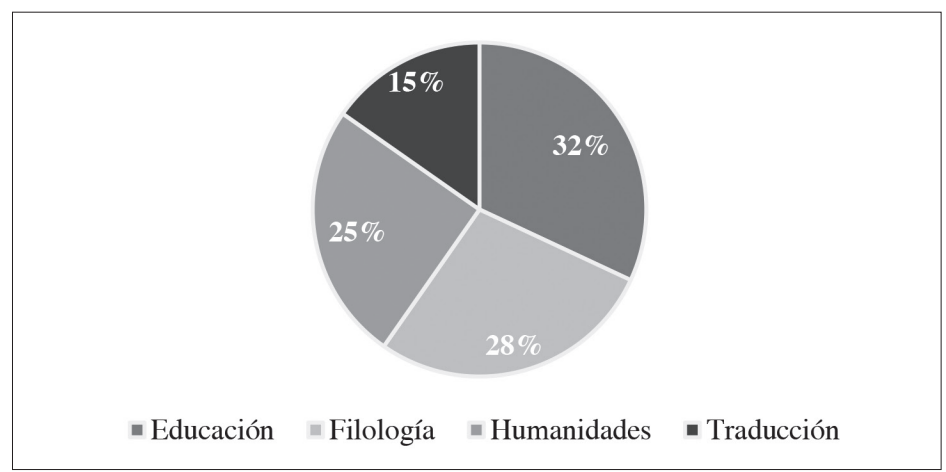

Gráfico 1. Formación en curso de las personas encuestadas.

Como se puede apreciar en el Gráfico 1, el porcentaje de participación ha sido similar entre todos los grados universitarios, aunque para el ámbito de Traducción se observa una menor cantidad de respuestas. Aun así, los resultados obtenidos son equitativos y muestran fielmente las opiniones de todos los encuestados. 
Por lo que se refiere a los datos personales (bloque 2), el promedio de edad de las personas que han respondido la encuesta es de 20.6. La mayoría oscilan entre los 18 y 19 años, aun así, la edad máxima registrada es de 35. El género que abunda es el femenino, ya que 108 respuestas pertenecen a este género, a diferencia de las 39 que se han registrado por el género masculino. Si se tiene en cuenta que, desde el punto de vista de las estadísticas oficiales, las mujeres poseen una mayor presencia en estas titulaciones, la diferencia de género está justificada. Asimismo, dichos datos no alteran el resultado de la encuesta, dado que no es una información significativa para la presente investigación.

Es importante destacar el lugar de nacimiento de los participantes. De las 147 personas, 11 nacieron en el extranjero (China, Argentina, Perú, Canadá, Ecuador, Chile y Alemania) y 4 en otras regiones de España (Valencia, Baleares y Aragón). El resto son originarias de Cataluña, la mayoría de ellos nacidos en la provincia de Barcelona, aunque también se encuentran casos de Tarragona, Lleida y Girona. Aun así, solo 4 han ubicado su residencia habitual fuera de Cataluña y, por tanto, la han situado en las ciudades de Mallorca, Valencia, Bruselas y Berlín.

Los resultados de las preguntas relacionadas con el uso de las lenguas (bloque 3) señalan que, a pesar del lugar de origen, solo el $3 \%$ de los encuestados tienen como lengua materna, según se muestra en el Gráfico 2, otra u otras lenguas que no son el español y/o el catalán:

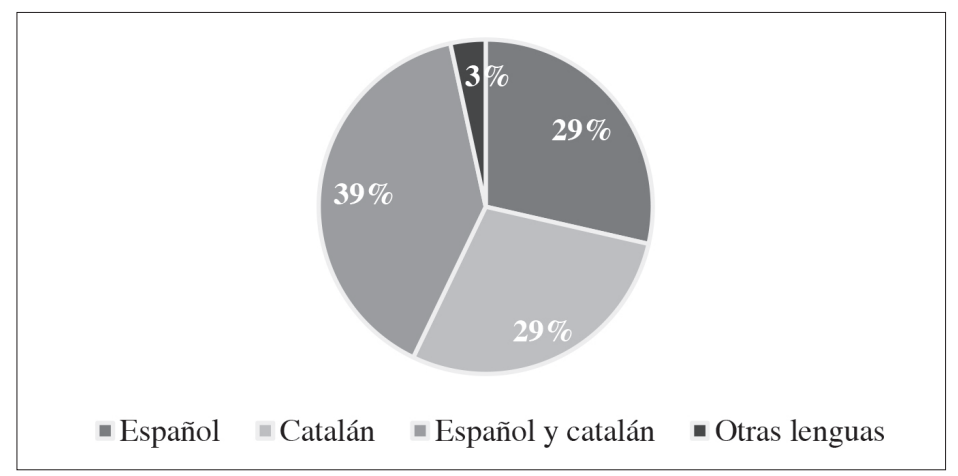

Gráfico 2. Lengua materna de los encuestados.

La mayoría, el $39 \%$ de los casos, reconocen tener como lengua materna el español y el catalán, seguido de dos porcentajes idénticos (29\%) que priorizan, respectivamente, uno de los dos idiomas. En este sentido, teniendo en cuenta los resultados acerca del lugar de origen y la residencia habitual, la fiabilidad de los datos obtenidos es de un $97 \%$, lo que favorece que todos sean usuarios potenciales de diccionarios bilingües español-catalán. 


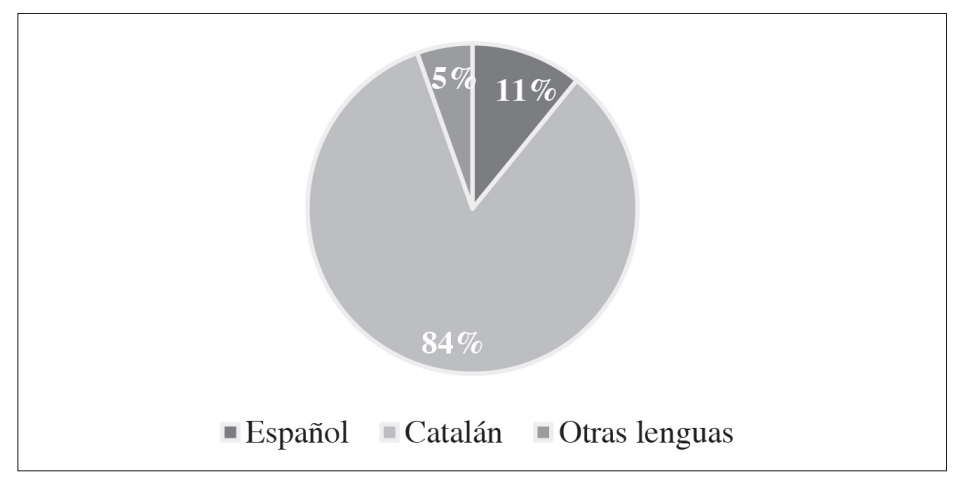

Gráfico 3. Lengua vehicular en la enseñanza básica.

La lengua utilizada en la formación básica es otro de los puntos a destacar. La mayor parte de los hablantes nacidos y formados en Cataluña pueden considerarse bilingües, según el grado de conocimiento de las lenguas y su uso. El modelo de inmersión lingüística en las escuelas e institutos a favor del catalán ha hecho crecer esta situación de bilingüismo, sobre todo entre los más jóvenes, quienes, a pesar de la lengua vehicular empleada en la formación básica, como se evidencia en los Gráficos 3 y 4, siguen haciendo un uso alterno de los dos idiomas en las situaciones más informales:

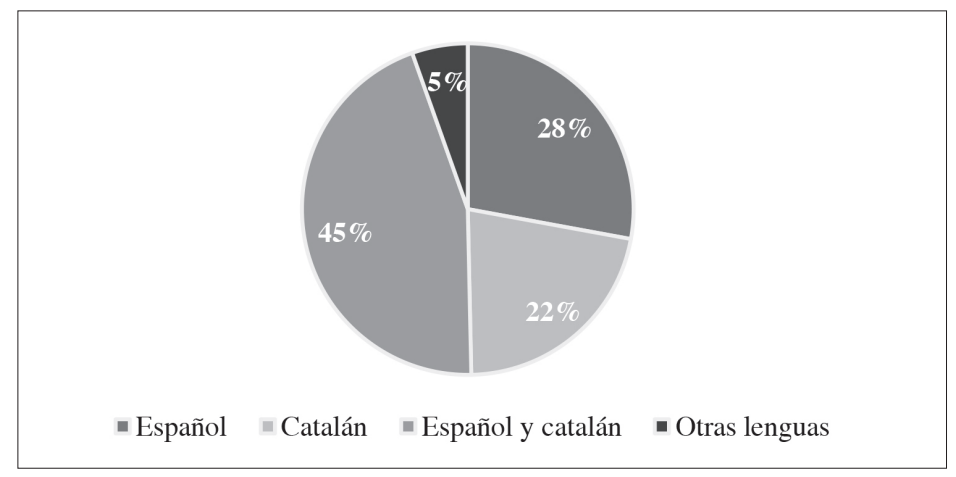

Gráfico 4. Lengua habitual (familia, amigos, entre otros).

Como consecuencia de todo lo explicado hasta este momento, analizar la concepción y el uso que poseen estas personas acerca de los diccionarios bilingües español-catalán (bloque 4) puede resultar de gran utilidad para los investigadores y lexicógrafos, como punto de partida para la reflexión y posterior aplicación en futuras obras de la información obtenida. 
La primera pregunta relacionada con el uso de estos diccionarios indica que el $85 \%$ de los encuestados, frente al $15 \%$ restante, han consultado en alguna ocasión una obra bilingüe en estas dos lenguas. Concretamente, tal y como refleja el Gráfico 5, las respuestas afirmativas se han dividido en sí, a menudo (31\%) y sí, en pocas ocasiones ( $54 \%$ ), lo cual se encuentra dentro de lo esperado si se tiene en consideración que la mayoría son conocedores de las dos lenguas. Por tanto, un diccionario bilingüe general solo se utiliza en casos concretos para la comprensión o producción de textos, a lo que se hará referencia más adelante.

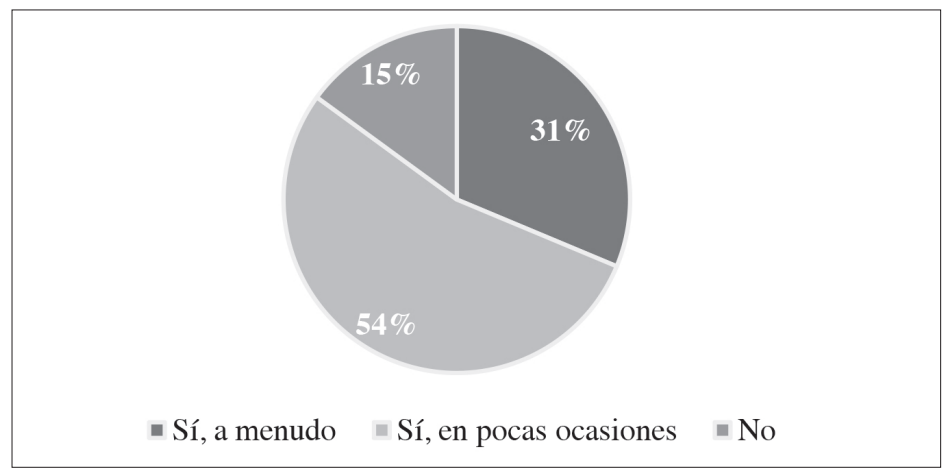

Gráfico 5. ¿Utilizas/has utilizado algún diccionario bilingüe español-catalán?

Sobre lo expuesto anteriormente, también se ha preguntado acerca de la utilidad de estos diccionarios. Los resultados ponen de manifiesto que la percepción general es positiva (el $73 \%$ del total, dividido en dos respuestas afirmativas, como se puede observar en el Gráfico 6), aunque, según se puede comprobar en las justificaciones que emiten, más de la mitad de los participantes consideran que hay aspectos a mejorar, como las traducciones que se proponen, la velocidad con la que se realiza la consulta, la renovación de los datos, la información que contienen las palabras o unidades recogidas, la claridad de los resultados, la fiabilidad de los datos, la cantidad de los ejemplos y su veracidad (que sean reales). Cabe resaltar que el $27 \%$ de las respuestas son negativas, lo cual significa que una tercera parte de las personas encuestadas no consideran que sean útiles tal y como están concebidos actualmente. 


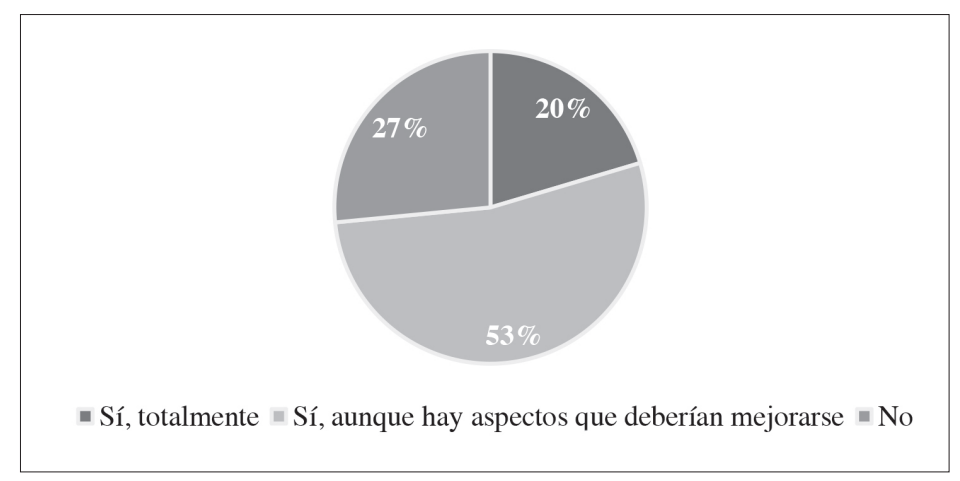

Gráfico 6. ¿Resultan útiles los diccionarios bilingües?

Las preguntas que más abundan son las que están vinculadas con los posibles usos y funciones de un diccionario electrónico de fraseología bilingüe españolcatalán (bloque 5), ya que es la propuesta que se planteará en futuros estudios. Para ello, se ha consultado a los participantes qué tipo de obra utilizarían a la hora de buscar el equivalente de la UF en la otra lengua (español o catalán). Las respuestas se recogen en el Gráfico 7:

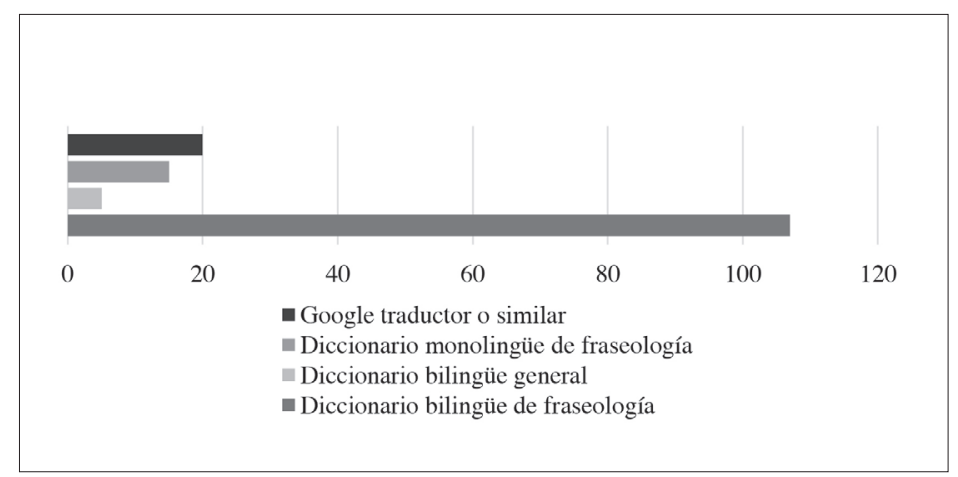

Gráfico 7. Tipo de diccionario que usarían los encuestados para buscar el equivalente de una UF.

Parece evidente que la mayor parte de las personas consideran que emplearían un diccionario bilingüe de fraseología para encontrar el equivalente en la otra lengua; sin embargo, por delante de los diccionarios bilingües generales o, incluso, los monolingües de fraseología, los encuestados preferirían realizar la búsqueda 
en alguna de las aplicaciones que se hallan en internet dedicadas a traducir. Esto resulta curioso si se tiene en cuenta que este tipo de programas no suelen proporcionar un equivalente propio de la otra lengua, sino que en la mayoría de los casos la traducción se lleva a cabo de manera literal.

La información que se acaba de aportar estaría en consonancia con la pregunta que se muestra en el Gráfico 8, donde los participantes debían seleccionar el tipo de formato que preferirían a la hora de consultar un diccionario bilingüe de fraseología. El 87 \% de los encuestados está a favor del formato electrónico, mientras que un $12 \%$ defiende su uso en papel. El $1 \%$ restante señala que «depende de la ocasión».

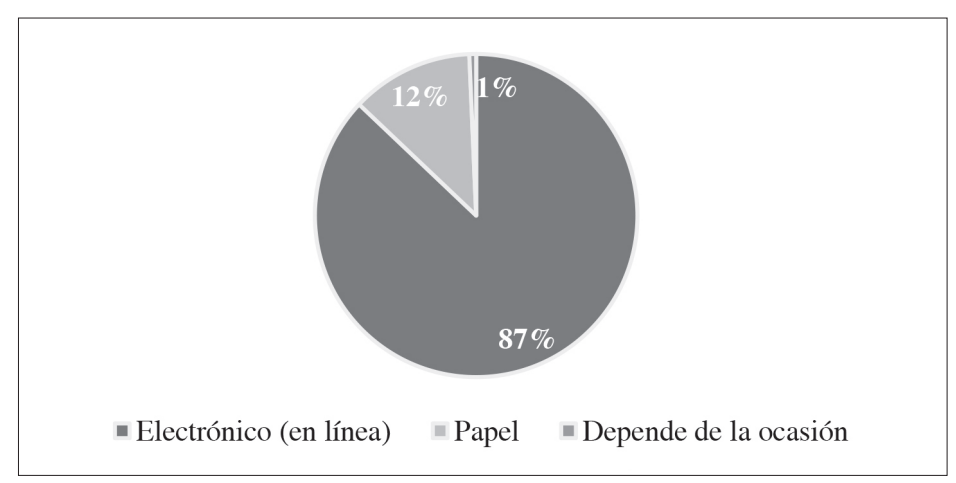

Gráfico 8. Preferencia de formato.

De cualquier modo, la mayoría (el $98 \%$ ) considera que sería necesario disponer de un diccionario electrónico de fraseología bilingüe español-catalán, tal y como se especifica en el Gráfico 9. Algunas de las justificaciones que aportan los participantes son las siguientes:

A. Actualmente es más habitual consultar recursos en este formato.

B. Este tipo de diccionarios sería útil para conocer cómo se dice en la otra lengua una expresión que no se puede traducir literalmente.

C. No existe aún un diccionario de este tipo.

D. La mayoría de las obras actuales no proporcionan un equivalente adecuado.

E. Un diccionario fraseológico bilingüe sería práctico y necesario para evitar las interferencias entre las dos lenguas implicadas.

F. Facilitaría el trabajo de los traductores. 


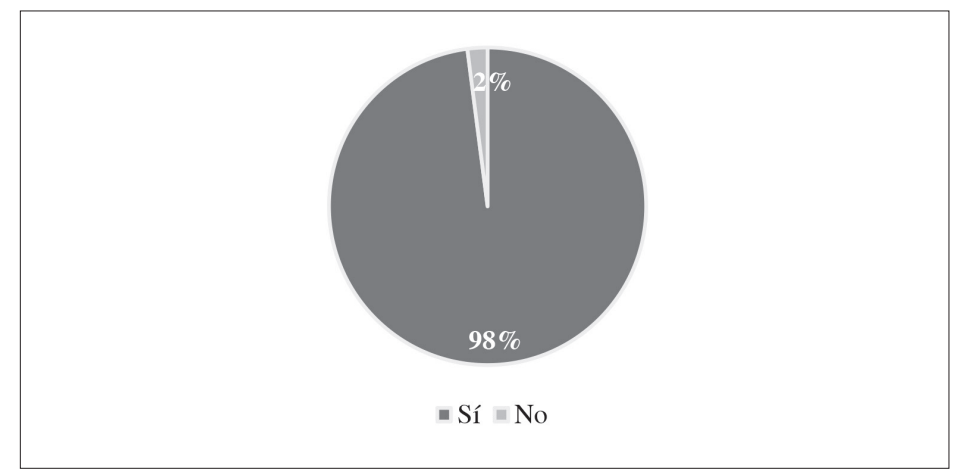

Gráfico 9. ¿Crees que sería necesario disponer de un diccionario electrónico de fraseología bilingüe español-catalán?

En cuanto a los rasgos que debería poseer un diccionario de este tipo, los encuestados valoran, sobre todo, la fiabilidad y la claridad de los datos presentados, es decir, que las unidades recogidas en la obra, además de presentarse de manera útil, estén documentadas de modo que no se traten de expresiones inventadas por parte del lexicógrafo (véase el Gráfico 10). Con una alta puntuación también destacan la actualización de los datos y la rapidez de la consulta, como dos características importantes para tener en cuenta a la hora de confeccionar un diccionario. Esto es razonable si se tiene en cuenta que algunas de las obras lexicográficas existentes en la actualidad no renuevan la información que recogen. El dinamismo y la personalización de los datos son otros de los rasgos que adquieren importancia cuando se accede a un diccionario, por este motivo han recibido una puntuación elevada. Por último, parece que los participantes consideran que los enlaces externos son el aspecto menos relevante, posiblemente porque, aunque suponga un añadido al diccionario, no determina su contenido.

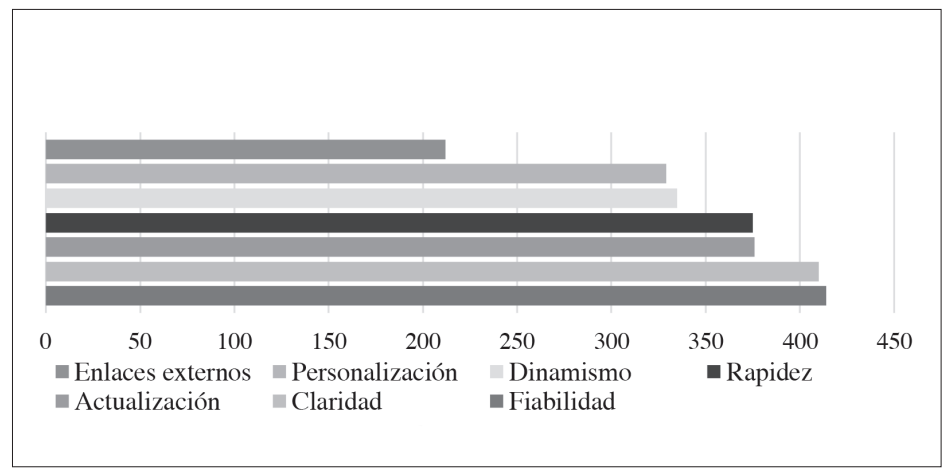

Gráfico 10. Características que debería poseer un diccionario electrónico de fraseología bilingüe español-catalán. 
Las respuestas que se acaban de presentar quedan totalmente justificadas a partir de la información extraída de las dos preguntas registradas en los Gráficos 11 y 12 que se indicarán a continuación. La mayoría de los participantes no conoce ningún diccionario de fraseología bilingüe español-catalán ni en papel ni en formato electrónico, aunque algunos de ellos responden afirmativamente a la pregunta. Estos últimos casos no se pueden considerar como respuestas válidas, puesto que el encuestado, cuando señala el nombre o la descripción del diccionario que conoce, indica o bien una obra bilingüe general o bien un diccionario monolingüe de fraseología.

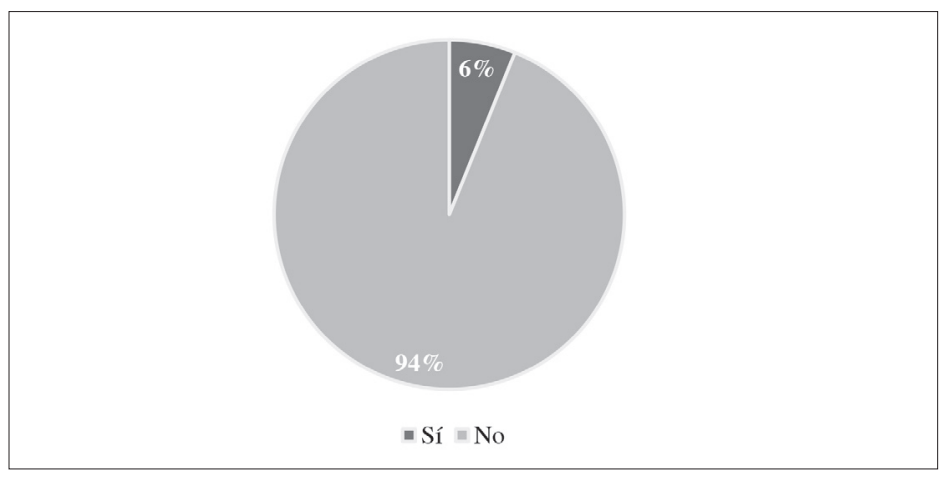

Gráfico 11. ¿Conoces alguno en papel?

Lo mismo ocurre con el formato electrónico: los que responden afirmativamente a la pregunta lo hacen señalando algunos recursos como el diccionario WordReference o Google, por lo que tampoco pueden considerarse respuestas fiables. Esto, además, confirma lo que ya advierten otros autores cuando señalan que existe una falta de unanimidad terminológica y conceptual a la hora de indicar los tipos de soporte en los que puede registrarse una obra. Por este motivo, es nece-

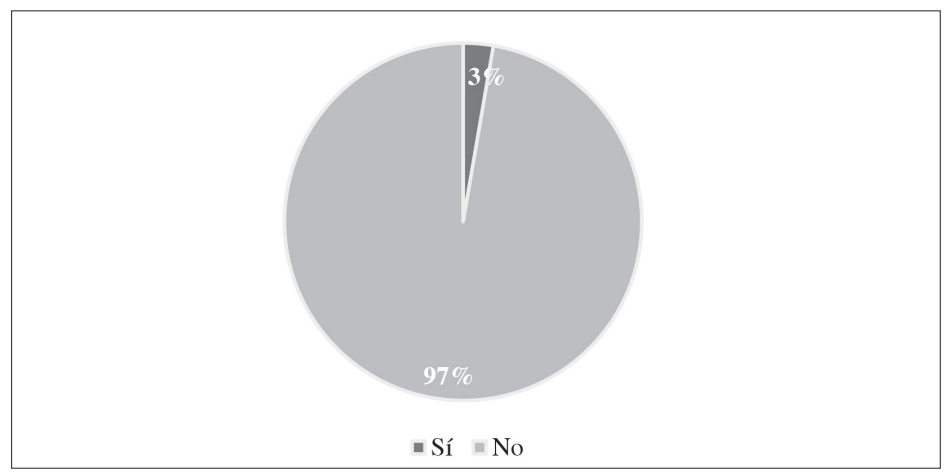

Gráfico 12. ¿Conoces alguno en formato electrónico? 
sario insistir en que el adjetivo electrónico no es sinónimo de digitalizado o en línea, aunque sí que se pueden llegar a combinar algunos de ellos según el lugar donde se ubique la obra: es posible encontrar un diccionario electrónico en línea ${ }^{5}$.

En relación con lo anterior, los encuestados han valorado, también, qué otro tipo de información (si lo creen conveniente) debería recogerse en un diccionario electrónico de fraseología bilingüe español-catalán además del equivalente. Tal y como aparece en el Gráfico 13, el significado es lo que destaca por encima del resto de informaciones. Esto demuestra que, a pesar de que tradicionalmente un diccionario bilingüe se concibe como una obra solo de consulta para conocer cómo se traduce en la otra lengua la palabra o expresión, los posibles usuarios señalan la importancia de poder incluir otras informaciones que ayuden a conocer mejor los datos presentados. Los ejemplos también son altamente valorados, puesto que recogen las evidencias de uso de las UFS. Un dato interesante es la puntuación otorgada al concepto como cuestión importante en una obra de este tipo. Los participantes lo sitúan en tercera posición por delante de la marcación, lo cual es llamativo. Por debajo se encuentra la información uso y, finalmente, las diferentes marcas, siendo la del registro la mejor valorada. A diferencia de los anteriores, una pequeña cantidad de encuestados considera que no es necesario incluir más información en un diccionario de esta tipología.

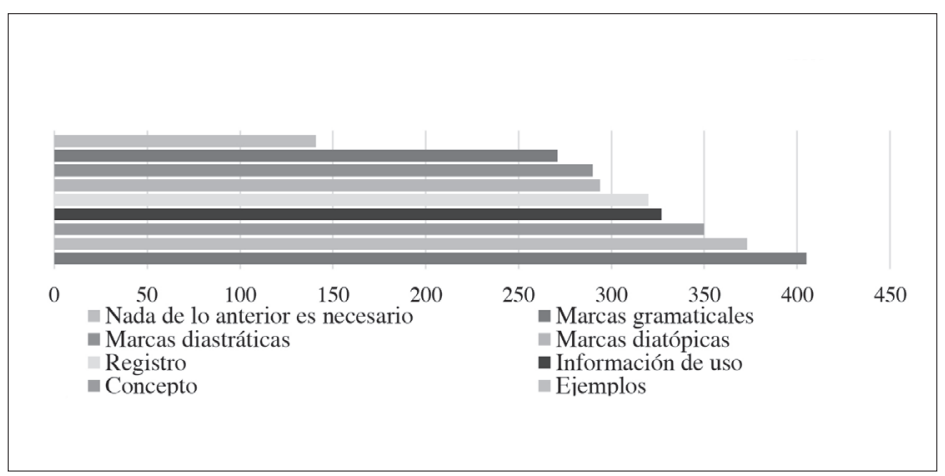

Gráfico 13. Información que debería aportar un diccionario electrónico de fraseología bilingüe español-catalán.

Es importante señalar, como ya se ha comentado anteriormente, las posibles funciones de un diccionario: comprensión (pasiva) o producción (activa). En el caso de la propuesta llevada a cabo en esta investigación, como se aprecia en el Gráfico 14, el $80 \%$ de las respuestas consideran adecuado concebir una obra de este tipo teniendo en cuenta las dos funciones, por lo que los posibles usuarios no solo utilizarían

${ }^{5}$ Véanse Águila (2009), Flinz (2010), Fuertes-Olivera y Bergenholtz (2011) y Fuertes-Olivera (2012) para ampliar la información. 
el diccionario para conocer cómo se dice una UF concreta en la otra lengua, sino también para entender qué significa y, de este modo, reforzar la comprensión. Sin embargo, los que han apostado por responder a una de las dos funciones, dan mayor importancia a la producción, lo que iría en consonancia con el perfil lingüístico y académico de los participantes, ya que le otorgan más valor a cómo expresar adecuadamente una UF en la otra lengua teniendo en cuenta los fenómenos de contacto que pueden producirse por el hecho de ser bilingües.

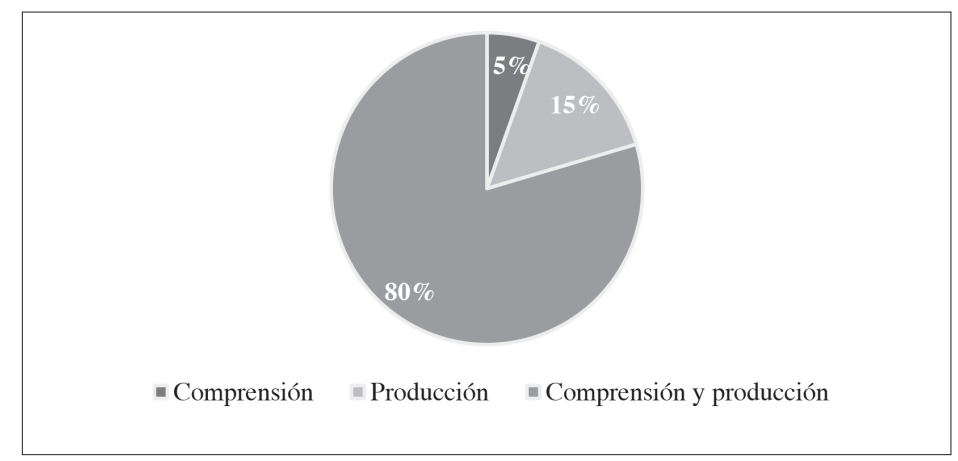

Gráfico 14. ¿Para qué utilizarías un diccionario de este tipo?

En definitiva, los participantes señalan que sería adecuado crear un diccionario de este tipo con el fin de proporcionar más información acerca de las UFS a los hablantes bilingües o aprendices de alguna de las dos lenguas.

La última pregunta, registrada en el Gráfico 15, está relacionada con la posibilidad de realizar distintos tipos de búsqueda, con la finalidad de que el usuario pueda concretar y personalizar la información que desea recibir por parte de la aplicación. El resultado ha sido claro: el $83 \%$ de los encuestados creen oportuno añadir diferentes opciones de búsqueda, en detrimento del $17 \%$ que no lo considera necesario.

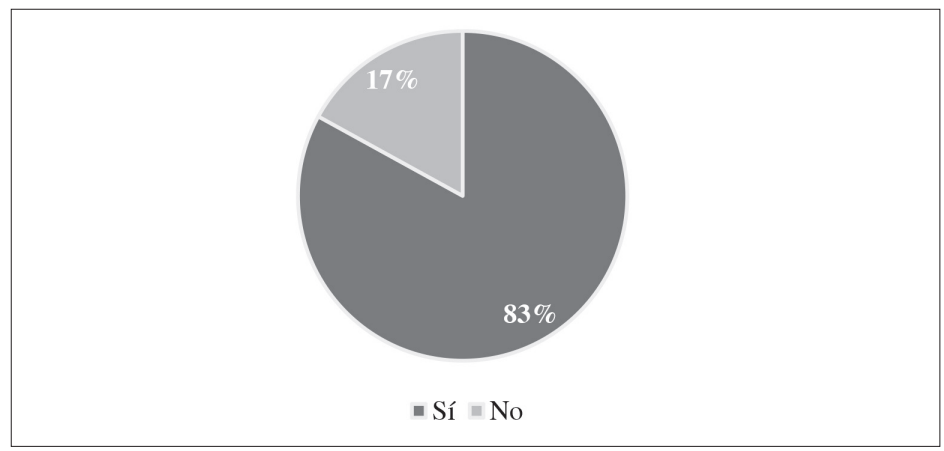

Gráfico 15. ¿Un diccionario con estas características debería permitir otro tipo de búsquedas? 
Asimismo, en el apartado de Otros aspectos a tener en cuenta, donde el encuestado ha podido dar su opinión libremente, destacan las siguientes informaciones:

A. Crear un diseño intuitivo que facilite el manejo y la accesibilidad a los datos.

B. Mayor calidad de las traducciones y, sobre todo, que la información proporcionada sea útil.

C. Incorporar un sistema de búsqueda que permita encontrar un fraseologismo con tan solo escribir una parte de él.

D. Poner énfasis en aquellas UFS que son difíciles de encontrar en la otra lengua por el hecho de ser equivalentes parciales o, incluso, nulos.

E. Acceder de manera gratuita. Esto último depende, sobre todo, de la financiación pública que puedan otorgarse a los proyectos que se están desarrollando en torno a este tipo de obras.

En suma, los resultados de la encuesta demuestran la necesidad de elaborar un diccionario electrónico de fraseología bilingüe español-catalán, puesto que no existe una obra que recoja todas las características mencionadas en este apartado. Asimismo, para poder llegar a cubrir el conjunto de las necesidades destacadas por los encuestados es conveniente tener en cuenta el servicio que un diccionario de este tipo otorgaría a los usuarios potenciales.

\section{CONCLUSIONES}

En este artículo se ha reflexionado en torno a las necesidades de crear un diccionario electrónico de fraseología bilingüe español-catalán. Se han constatado, mediante las encuestas llevadas a cabo, las preferencias formales y de contenido que debería presentar una obra de estas características, lo que corrobora la importancia de conocer la opinión de los posibles usuarios con la ayuda de métodos experimentales como el que se ha ejecutado en este estudio.

La encuesta se ha preparado mediante Google Formularios y tiene como objetivo conocer la percepción de los participantes acerca de los diccionarios bilingües, en general, y del posible uso de un diccionario de fraseología bilingüe para las dos lenguas aquí objeto de estudio, en particular. Como se ha podido comprobar, las preguntas se han dividido en grupos según el tipo de información que se desea recoger. Por un lado, ha sido importante tener en cuenta el perfil lingüístico y académico de las personas participantes, ya que de este modo se han podido extraer conclusiones más determinantes. Por otro, partir de lo general (diccionario bilingüe) para llegar a lo concreto (diccionario propuesto) ha sido un procedimiento 
útil que ha permitido guiar al encuestado y proporcionarle las herramientas contextuales suficientes para entender de mejor modo las preguntas formuladas.

La utilidad de esta metodología es elevada si se tiene en cuenta que la mayoría de las propuestas de diccionario no se basan en las aportaciones de los usuarios potenciales, sino en estudios precedentes que a su vez proceden de otros anteriores. En este trabajo se ha defendido la puesta en práctica de una metodología experimental como punto de partida para elaborar una futura obra de consulta pública.

A partir de las 22 preguntas realizadas, los resultados expresan el interés que muestran los encuestados ante la posibilidad de poder tener a su disposición un diccionario de este tipo, ya que, como algunos de ellos indican, no se encuentra una obra similar a disposición de los usuarios. Es evidente, además, que el formato preferido para la consulta es el electrónico, debido a que resulta más cómodo y la búsqueda es más rápida.

La gran mayoría de los posibles interesados señalan la utilidad limitada que presentan los diccionarios bilingües actuales. En relación con esto determinan que se deberían mejorar algunas de sus características como, por ejemplo, la fiabilidad de los datos aportados y la claridad de la información. Se valora con una alta puntuación la rapidez y la actualización de los datos, así como su dinamismo y la personalización de las búsquedas y, en menor grado, la conexión a enlaces externos.

Son igual de importantes e interesantes los resultados que se han obtenido acerca del tipo de información que debería contener un diccionario electrónico de fraseología bilingüe en español y catalán. La mayor parte de los participantes considera que el significado, los ejemplos y, curiosamente, los conceptos que subyacen a las UFS son algunos de los aspectos que deberían incorporarse en una obra de este tipo.

En definitiva, se ha demostrado la necesidad de concebir cuanto antes una obra que reúna, por un lado, los avances en la lexicografía electrónica y, por otro, los resultados de los estudios fraseológicos contrastivos en español y catalán, hasta ahora apenas inexistentes. Por tanto, en futuras investigaciones sería pertinente aplicar los datos obtenidos en la encuesta para proponer un diccionario electrónico de fraseología bilingüe que, sin duda, se convertiría en un referente para la fraseología y la fraseografía de estas dos lenguas.

\section{REFERENCIAS}

Águila Escobar, Gonzalo. (2009). Los diccionarios electrónicos del español. Madrid: Arco/Libros.

Azorín Fernández, Dolores. (2000a). “¿Para qué usan el diccionario los escolares? Reflexiones a propósito de una encuesta”. En Ruhstaller, S., y Prado Aragonés, 
J. (eds.). Tendencias en la investigación lexicográfica del español. El diccionario como objeto de estudio lingüistico y didáctico. Huelva: Universidad de Huelva. pp. 75-100.

Azorín Fernández, Dolores. (2000b). Los diccionarios didácticos del español desde la perspectiva de sus destinatarios, ELUA 4, pp. 19-44.

Climent de Benito, Jaime. (2003). "Sobre una encuesta: los diccionarios castellanovalenciano, valenciano-castellano y el estudiante de Secundaria”, Actas del Congreso Internacional "La Lengua, la Academia, lo Popular, los Clásicos, los Contemporáneos... ”. Vol. II (pp. 505-517), Alicante: Universidad de Alicante.

Corpas Pastor, Gloria y Morvay, Károly. (2002). "Los estudios de fraseología y fraseografía en la Península Ibérica". Annales Universitatis Scientiarum Budapestinensis de Rolando Eötvös Nominatae. Sectio Lingüística, vol. 25, pp. 165182.

D62 = VV.AA. (2003). Diccionari 62 doble català-castellà $i$ castellà-català. Barcelona: Edicions 62.

DCC = Enciclopèdia Catalana. (2006). Diccionari català-castellà (versión en papel). Barcelona: Enciclopèdia Catalana.

DFDEA = Seco, Manuel, Olimpia Andrés y Gabino Ramos. (2004). Diccionario fraseológico documentado del español actual. Madrid: Aguilar lexicografía.

Flinz, Carolina. (2010). "DIL': a German-Italian online specialized dictionary of linguistics". En Sylviane Granger y Magali Paquot (eds.) eLexicography in the $21^{s t}$ Century: New Challenges, New Applications (67-76). Bélgica: Presses universitaires de Louvain.

Fuertes-Olivera, Pedro. (2012). La lexicografía de internet: el 'Diccionario inglésespañol de contabilidad', Círculo de Lingüistica Aplicada a la Comunicación 52: 21-56. Disponible en línea <http://www.ucm.es/info/circulo/no52/fuertes.pdf>. Fuertes-Olivera, Pedro A. y Bergenholtz, Henning. (2011). "Introduction: The Construction of Internet Dictionaries". En Pedro A. Fuertes-Olivera y Henning Bergenholtz (eds.) e-Lexicography. The Internet, Digital Initiatives and Lexicography, (1-16). London/New York, Continuum.

García Rodríguez, Joseph. (2016) La evolución de la fraseología española en los diccionarios bilingües, e-AESLA 2, pp. 383-392. Disponible en línea <https:// cvc.cervantes.es/lengua/eaesla/pdf/02/35.pdf>.

García Rodríguez, Joseph. (en prensa, 2019). "La fraseología de los colores en el contexto bilingüe español-catalán”. En Konecny, Christine, Autelli, Erica, Abel, Andrea y Zanasi, Lorenzo (eds.). Lexemkombinationen und typisierte Rede im mehrsprachigen Kontext. 2 vols. Tübingen: Stauffenburg [Stauffenburg Linguistik].

García Rodríguez, Joseph. (2020). La fraseología del español y el catalán: semántica cognitiva, simbolismo y contrastividad (Studien zur romanischen 
Sprachwissenschaft und interkulturellen Kommunikation. Heraugegeben von Gerd Wotjak). Frankfurt am Main: Peter Lang.

GDUEsA = VV.AA. (2001). Gran diccionario de uso del español actual. Madrid: SGEL.

Meijs, Willem (1990). Morphology and word-formation in a machine-readable dictionary: Problems and possibilities. Folia Linguistica: Acta Societatis Linguisticae Europaeae, 24.1-2, pp. 45-71.

MOLL = Moll, Francesc de B. (1980). Diccionari F. B. Moll català-castellà $i$ castellàcatalà. Palma de Mallorca: Editorial Moll.

Müller-Spitzer, Carolin et al. (2011). "What makes a good online dictionary? Empirical insights from an interdisciplinary research project". En Iztok K. (ed. lit.), Karmen K. (ed. lit.) Electronic lexicography in the 21st century: New Applications for New Users: Proceedings of eLex 2011 (pp. 203-208) Liubliana [Eslovenia]: Trojina, Institute for Applied Slovene Studies.

Prat Sabater, Marta. (2015). "Interferències lingüístiques entre català i castellà en el context universitari. L'expressió escrita", Estudis de llengua i literatura catalanes/ LXIX. Miscel-lània Jordi Bruguera, 3. Publicacions de l'Abadia de Montserrat, pp. 173-205.

Sala Caja, Alicia. (2015). "Actitudes de estudiantes y profesores hacia los diccionarios electrónicos portátiles”. En ASELE, La Enseñanza de ELE centrada en el alumno. Getafe: Universidad Carlos III.

\section{ANEXO}

\section{ENCUESTA}

1. Género.

2. Edad.

3. Lugar de nacimiento.

4. Lugar de residencia habitual.

5. Formación (cursada o actualmente cursando).

6. Lengua materna.

Español

Catalán

Español y catalán

Otro: 
7. Lengua vehicular en la enseñanza básica (primaria y secundaria). Español

Catalán

Otro:

8. Lengua habitual (familia, amigos...).

Español

Catalán

Español y catalán

Otro:

9. ¿Has utilizado/utilizas algún diccionario bilingüe español-catalán?

Sí, a menudo

Sí, en pocas ocasiones

No

Otro:

10. Tal y como están concebidos este tipo de diccionarios, ¿crees que resultan útiles?

Sí, totalmente

Sí, aunque hay aspectos que deberían mejorarse

No

Otro:

11. Señala alguno de los aspectos a mejorar en estas obras, si es que así lo has considerado en la pregunta anterior.

12. Si tuvieras que consultar cómo se dice en catalán la expresión llevarse el gato al agua, ¿qué diccionario o recurso utilizarías?

- Diccionario monolingüe

- Diccionario bilingüe de frases hechas, refranes, etc.

- Diccionario bilingüe general

- Diccionario monolingüe de frases hechas, etc.

- Google traductor o similar

- Otro:

13. ¿En qué formato preferirías realizar la consulta?

- Papel

- Electrónico (en línea)

- Otro: 
14. ¿Crees que sería necesario disponer de un diccionario ELECTRÓNI$\mathrm{CO}$ de fraseología (frases hechas, refranes...) bilingüe español-catalán?

- Sí

- No

- Otro:

15. Justifica brevemente por qué crees (o no) que sería necesario tener a nuestro alcance un diccionario ELECTRÓNICO de fraseología bilingüe español-catalán.

16. La fraseología es la disciplina que estudia las frases hechas, los refranes, etc. En tu opinión, ¿qué rasgos debería poseer un diccionario ELECTRÓNICO de fraseología bilingüe español-catalán? (valora del 0 -nada- al 3 -mucho- la importancia de las siguientes características).

\begin{tabular}{|l|l|l|l|l|}
\hline Características & 0 & 1 & 2 & 3 \\
\hline La claridad de la información & & & & \\
\hline La fiabilidad de los datos & & & & \\
\hline La rapidez de la consulta & & & & \\
\hline La actualización de los datos & & & & \\
\hline La personalización de las búsquedas & & & & \\
\hline El dinamismo de los datos & & & & \\
\hline La conexión mediante & & & & \\
\hline Hipervínculos con otros recursos en línea & & & & \\
\hline
\end{tabular}

17. ¿Conoces algún diccionario de fraseología bilingüe español-catalán en PAPEL? (Sí o no. Si es que sí, indica cuál).

18. ¿Conoces algún diccionario de fraseología bilingüe español-catalán en formato ELECTRÓNICO? (Sí o no. Si es que sí, indica cuál).

19. Además del equivalente en la otra lengua (ej.: en abril, aguas mil - a l'abril, aigües mil), ¿crees que un diccionario ELECTRÓNICO de fraseología bilingüe debería aportar más información? (Valora del 0 al 3 la importancia). 


\begin{tabular}{|l|l|l|l|l|}
\hline Tipo de información & 0 & 1 & 2 & 3 \\
\hline Significado & & & & \\
\hline Ejemplos & & & & \\
\hline Marcas gramaticales (ej.: locución verbal) & & & & \\
\hline Marcas de registro (ej.: coloquial) & & & & \\
\hline Marcas diastráticas (ej.: jerga juvenil) & & & & \\
\hline Marcas diatópicas (ej.: Cataluña) & & & & \\
\hline $\begin{array}{l}\text { Información de uso (ej.: esta expresión se suele } \\
\text { construir con el verbo estar) }\end{array}$ & & & & \\
\hline Hipervínculos con otros recursos en línea & & & & \\
\hline $\begin{array}{l}\text { En estos tipos de diccionario solo es importante el } \\
\text { equivalente que se propone en la otra lengua, lo de- } \\
\text { más no es necesario }\end{array}$ & & & & \\
\hline $\begin{array}{l}\text { El concepto en que se ubica la expresión (ej.: } \\
\text { echar fuego por los ojos - tirar foc pels queixals } \rightarrow \\
\text { concepto: IRA) }\end{array}$ & & & & \\
\hline
\end{tabular}

20. ¿Para qué utilizarías un diccionario ELECTRÓNICO de fraseología español-catalán con las características que se han ido mencionando anteriormente?

\begin{tabular}{|l|c|}
\hline Usos & $\mathrm{X}$ \\
\hline Para entender el significado de una expresión en la otra lengua (comprensión) & \\
\hline $\begin{array}{l}\text { Para saber cómo se dice y cómo se utiliza la expresión en la otra lengua (pro- } \\
\text { ducción de textos) }\end{array}$ & \\
\hline Para los dos casos anteriores (comprensión y producción) & \\
\hline Otro: & \\
\hline
\end{tabular}

21. Además de la búsqueda del equivalente en la otra lengua, ¿crees que un diccionario ELECTRÓNICO de estas características debería permitir otro tipo de búsquedas? (por marca, por concepto, etc.).

- Sí

- No

- Otro:

22. Como usuario de diccionarios, ¿qué otros aspectos no comentados anteriormente crees que deberían tenerse en cuenta a la hora de confeccionar un diccionario ELECTRÓNICO de fraseología bilingüe español-catalán? 